\title{
Robust Whispering-Gallery-Mode Microbubble Lasers from Colloidal Quantum Dots
}

Yue Wang, ${ }^{\dagger}$ Van Duong Ta, ${ }^{\dagger,}$ Kheng Swee Leck, ${ }^{\S}$ Beng Hau Ian Tan, ${ }^{\dagger}$ Zeng Wang, ${ }^{\dagger, \|}$ Tingchao He, ${ }^{\perp}$

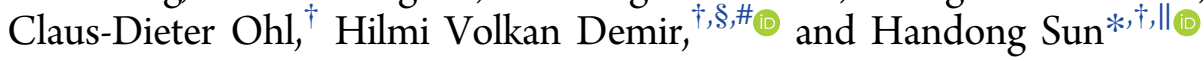

${ }^{\dagger}$ Division of Physics and Applied Physics, School of Physical and Mathematical Sciences, "Centre for Disruptive Photonic

Technologies (CDPT), School of Physical and Mathematical Sciences, Nanyang Technological University, Singapore 637371, Singapore

${ }^{\ddagger}$ Department of Physics, King’s College London, Strand, London, WC2R 2LS, United Kingdom

${ }^{\S}$ School of Electrical and Electronic Engineering, Luminous! Center of Excellence for Semiconductor Lighting and Displays, Nanyang Technological University, Nanyang Avenue, Singapore 639798, Singapore

${ }^{\perp}$ College of Physics Science and Technology, Shenzhen University, Shenzhen 518060, China

\# Department of Electrical and Electronics Engineering, Department of Physics, and UNAM-National Nanotechnology Research Center, Bilkent University, Bilkent, Ankara 06800, Turkey

\section{Supporting Information}

ABSTRACT: Microlasers hold great promise for the development of photonics and optoelectronics. Among the discovered optical gain materials, colloidal quantum dots (CQDs) have been recognized as the most appealing candidate due to the facile emission tunability and solution processability. However, to date, it is still challenging to develop CQD-based microlasers with low cost yet high performance. Moreover, the poor long-term stability of CQDs remains to be the most critical issue, which may block their laser aspirations. Herein, we developed a unique but generic approach to forming a novel type of a whispering-gallery-mode (WGM) microbubble laser from the hybrid CQD/poly(methyl methacrylate) (PMMA) nanocomposites. The formation mechanism of the microbubbles was unraveled by recording the drying process of the nanocomposite droplets. Interestingly, these microbubbles naturally serve as the high-quality WGM laser resonators. By simply changing the CQDs, the lasing emission can be tuned across the whole visible spectral range. Importantly, these microbubble lasers exhibit unprecedented long-term stability (over one year), sufficient for practical applications. As a proof-of-concept, the potential of water vapor sensing was demonstrated. Our results represent a significant advance in microlasers based on the advantageous CQDs and may offer new possibilities for photonics and optoelectronics.

KEYWORDS: Microlaser, quantum dot, microbubble, whispering-gallery-mode, nanocomposites

$M$ icrolasers hold great potential in a wide range of applications ranging from sensing to photonic integrated circuits. $^{1-3}$ Low-cost yet high-performance microlasers have always been pursued, which could offer new possibilities in photonics and optoelectronics. ${ }^{4-8}$ Since the first observation of stimulated emission, colloidal quantum dots (CQDs) have emerged as the most promising optical gain media due to the facile emission color tunability, potentially low and temperatureinsensitive gain threshold, and cost-effective solution processability. ${ }^{5-16}$ However, up until now, it is still challenging to develop CQD-based microlasers with low cost yet high performance. ${ }^{4,10,13}$

Moreover, CQDs are generally vulnerable to oxygen/moisture and will degrade upon exposure to air due to the existence of labile organic ligands. ${ }^{17-20}$ Consequently, it is highly desirable to encapsulate the CQDs with a protective matrix so as to attain robustness against the environment. To this end, researchers have attempted to incorporate the CQDs into inorganic sol-gel titania or silica, ${ }^{18,21,22}$ in which, however, complex ligand exchange is requisite and the photoluminescence quantum yield (PL QY) of the CQDs was largely compromised. Organic polymers, exemplified by poly(methyl methacrylate) (PMMA), have been recognized as the ideal matrix for CQDs due to the low cost, favorable mechanical flexibility, optical transparency across the full visible regime, and chemical/physical inertness. ${ }^{23,24}$ As a matter of fact, hybrid organic-inorganic CQD/PMMA nanocomposites have been exploited in various optoelectronic devices, such as active waveguides, solar concentrators, light-

Received: February 1, 2017

Revised: $\quad$ March 10, 2017

Published: March 13, 2017 


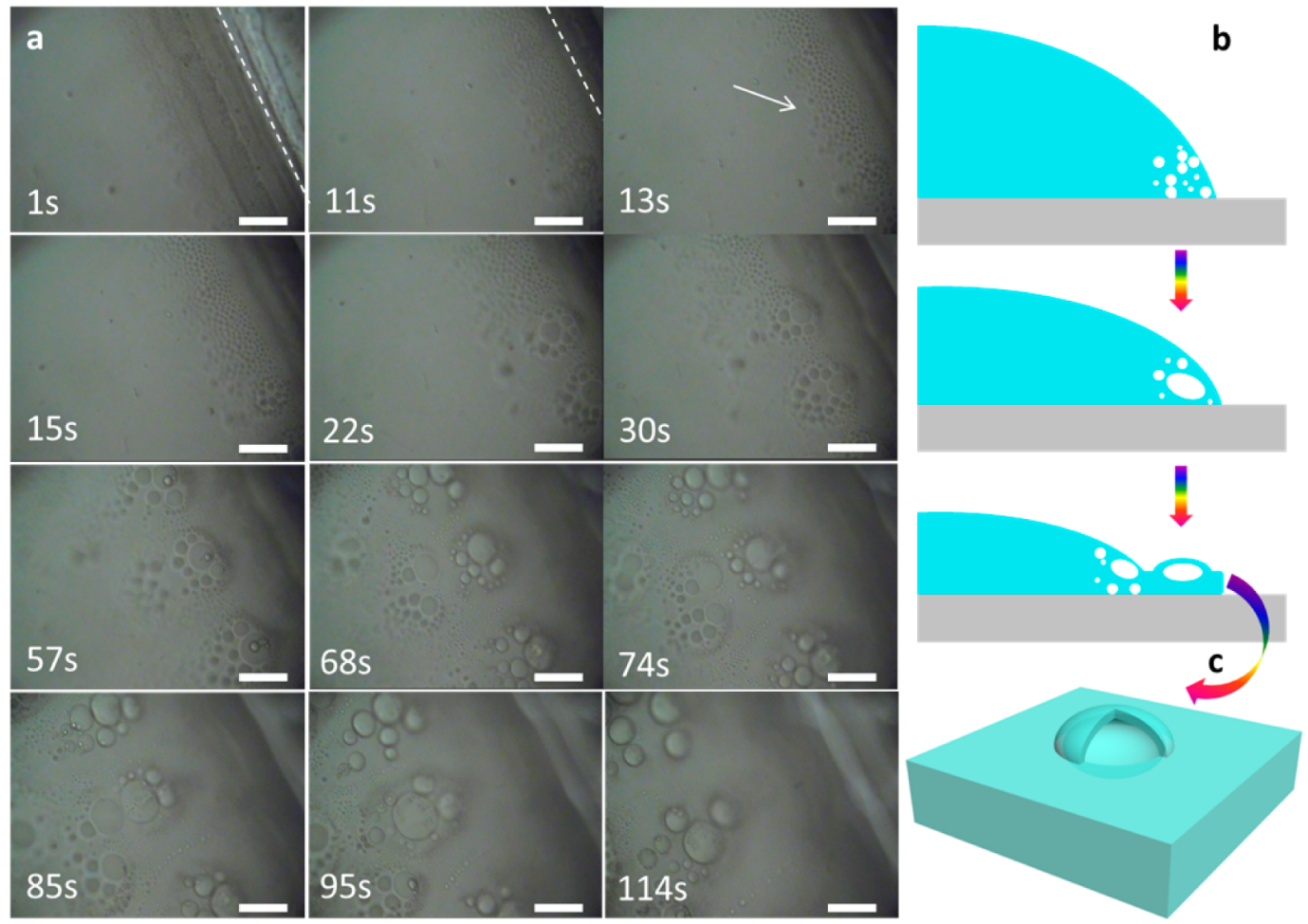

Figure 1. (a) Representative images during the drying process of CdZnS/ZnS CQD/PMMA (41 wt \%) nanocomposite droplet. The white dashed lines indicate the contact line. The white arrow shows the appearance of bubbles. All scale bars are $50 \mu \mathrm{m}$. (b) Schematic illustration of the formation of microbubbles during the drying process of CQD/PMMA nanocomposite droplet. (c) Schematic structure of the microbubble formed in the CQD/ PMMA nanocomposite film.

emitting diodes (LEDs), and displays. ${ }^{23-25}$ However, a typically low loading concentration $(<\sim 10 \%)$ of CQDs was used in order to obtain homogeneous nanocomposite films, which is certainly undesired for lasing aspirations. ${ }^{9,26}$

In recent years, whispering-gallery-mode (WGM) microlasers have garnered tremendous attention as a result of the small mode volume and high quality factor (Q-factor). ${ }^{27-32}$ Especially, the resonant wavelength of a WGM microlaser is so sensitive to the interaction of the evanescent field and the nearby environment that even a single nanoparticle can be detected. ${ }^{33-36}$ CQD-based WGM microlasers have been demonstrated by employing suitable templates to support the WGM resonance, such as spheres and toroids. ${ }^{13,17,37,38}$ Yet, a deliberately designed template with a smooth surface and favorable adhesion of CQDs onto the templates are big concerns, ${ }^{17}$ which necessitates the tough and time-consuming surface functionization. Moreover, the manipulation of the lasing modes becomes cumbersome, which can only be enabled by changing the templates. $^{17}$

Herein, a novel type of CQD-based WGM microlasers are realized from the highly concentrated CQD/PMMA ( 45 wt \%) nanocomposites. The formation of the microbubbles was explored by recording the drying process of the nanocomposite droplets and attributed to the synergetic effects of CQD-assisted bubbling and stabilizing by PMMA. Interestingly, these microbubbles naturally serve as high-quality WGM resonators, rendering low-threshold and high-Q microlasers. By simply changing the CQDs, the lasing emission can be tuned spanning blue, green, and red colors. The size of the microbubble and thus the longitudinal cavity modes can be facilely manipulated by tailoring the CQD concentration. Importantly, these microbubble lasers exhibit unprecedented long-term stability thanks to the packaging of PMMA, sufficient for practical applications. As a proof-of-concept, the potential of water vapor sensing was demonstrated. Our results establish a unique but generic approach toward cost-effective, easy mass productive, and highperformance CQD-based microlasers, which not only function well as an excellent platform to explore light-matter interaction but also infuse fresh blood into the development of practical microlasers.

Pursuing hybrid CQD/PMMA nanocomposites toward highly robust lasing media remains tempting as discussed above. In order to achieve sufficient optical gain, we tentatively increase the CQD concentration in the nanocomposites. Interestingly, as the concentration of the CQDs increases far above the typical value used for waveguides, LEDs and solar concentrators, domeshaped microbubbles will be self-assembled, as illustrated in the following. Herein, the blue-emitting $\mathrm{CdZnS} / \mathrm{ZnS}$ alloyed coreshell CQDs were adopted as the example since the favorable optical gain characteristics have been disclosed in our previous work. $^{10,26}$ To prepare the CdZnS/ZnS CQD/PMMA nanocomposite solution, PMMA was first dissolved in toluene with concentration of $10 \mathrm{wt} \%$ by ultrasonic for $2 \mathrm{~h}$. Then, the asprepared CdZnS/ZnS CQDs with concentration of $30 \mathrm{mg} / \mathrm{mL}$ in toluene was mixed with the PMMA stock solution in varied proportion. The mixed solution looked as clear as that of the pure solution and no precipitate was observed (Figure S1, Supporting Information (SI)). The absorption, PL spectra, and PL dynamics of the as-prepared CdZnS/ZnS CQD solution and the CdZnS/ $\mathrm{ZnS}$ CQD/PMMA mixed solution (volume ratio of 1:2 for PMMA-CQD solution) were found to be nearly identical (Figure S2, SI), which indicates that the introduction of PMMA does not strongly influence the optical properties of $\mathrm{CdZnS} / \mathrm{ZnS}$ CQDs. Similar phenomena have been previously observed in 
other kinds of CQDs and PMMA nanocomposites. ${ }^{23,24}$ Finally, the mixed CQD/PMMA solution was drop-casted onto a precleaned glass substrate to fabricate the solid nanocomposite film. Figure S3 in the SI shows the surface morphologies of the dried films with a varied CQD concentration ranging from 0 to $51 \%$ detected by optical microscopy. It is interesting to find that protuberant microbubbles, which are confirmed by scanning electron microscopy (SEM) (Figure S4, SI), revealing the hollow structure, are formed with the increase of CQD concentration in the nanocomposite. The size of the bubbles is closely related to the concentration of the CQDs varying from several micrometers for $\sim 25 \%$ to several tens of micrometers for $\sim 43 \%$. For higher CQD concentrations ( $>50 \%)$, the microbubbles tend to crack due to the existence of internal strain.

In order to unravel the origin of the bubbling, we performed several control experiments by recording the drying process of droplets of different compositions: (a) PMMA in toluene (Video S1, SI); (b) CQDs in toluene (Video S2, SI), and (c) CQD/ PMMA nanocomposite (41 wt \%) in toluene (Video S3, SI). The PMMA droplet evaporated without any observable bubbling at the contact line, ruling out PMMA as the origin of the bubbling. In contrast, it can be found that the evaporating CQD droplet produced tiny bubbles at the contact line, which merged into larger microbubbles. However, all of the bubbles dissolved into the solution over a time scale of $\tau \sim 1 \mathrm{~min}$. When a drop-casted CQD/PMMA droplet was deposited onto the substrate, tiny bubbles appeared at the contact line with the evaporation of the solvent (see representative frames in Figure 1a). The tiny bubbles grew with time and merged to form larger microbubbles. Notably, the microbubbles survived even after the contact line of the droplet had moved over it and dried completely, suggesting that the polymeric nature of the PMMA facilitates the bubble's stability.

Although we could not directly verify that the objects were bubbles, the experiments provide compelling evidence for their gaseous nature. First, it is unlikely that the objects seen here are droplets formed by the Ouzo effect, since this requires at least one of the liquids to be immiscible in order for nanodroplets of liquid to be formed; ${ }^{39}$ second, the objects shrink in toluene over a time scale consistent with a gas bubble. A gas bubble in liquid is known to obey the diffusion equation ${ }^{40,41}$ and shrink within a time scale $\tau \sim K a^{2} / 3 R T D,{ }^{41}$ where $K$ is the Henry's law constant, $a$ the initial bubble radius, $R$ the ideal gas constant, $T$ temperature, and $D$ the diffusion constant. Using $K \sim 10^{5} \mathrm{~J} /$ mol, $a=50 \times 10^{-6} \mathrm{~m}$, and $D \sim 10^{-9} \mathrm{~m}^{2} / \mathrm{s}$, ${ }^{40}$ we obtain a characteristic time scale of $\tau \sim 30 \mathrm{~s}$, in agreement with the experiment. Note that the theory is an underestimate since it does not take into account collective effects from neighboring bubbles which can increase the bubble lifetime further. ${ }^{42,43}$ Finally, the gaseous nature of the objects is also consistent with the stabilization of the objects with PMMA. The addition of $10 \%$ by weight polymeric PMMA to toluene raises the viscosity of the toluene solvent by about 2 orders of magnitude, ${ }^{44}$ and the diffusion constant is reduced by a proportionate amount by the Stokes-Einstein equation, ${ }^{44}$ leading to a 2 orders of magnitude increase in the bubble lifetime $\tau$. This increase in lifetime is more than enough to sustain the bubble for the full duration of the experiment. At equilibrium, all liquids contain some quantity of dissolved gas. However, the free energy of formation of a microbubble is not large enough for them to form spontaneously. While, for the CQDs, the small size and the hydrophobic surface nature ${ }^{2,6}$ make them energetically suitable as nuclei, significantly reducing the free energy barrier for the formation of a bubble on the CQD. ${ }^{45}$ Therefore, the CQDs serve as nanoscopic nuclei for the nucleation of bubbles. According to the nonequilibrium bubbling process, CQDs may accumulate in concentration around the bubbles. However, it is extremely challenging to precisely map the distribution of CQDs across the bubble, especially for the area near the inner wall, due to the unflatness and hollow structure of the sample. Leveraging on the synergetic effects of CQD-assisted bubbling and stabilizing by PMMA, the microbubbles are obtained. The schematic bubble formation process is depicted in Figure 1b. As a matter of fact, analogous nanobubbles or microbubbles that are unintentionally formed have been observed in previous reports, where such samples were deemed as defective. ${ }^{46}$

Thanks to the mechanical flexibility of the PMMA matrix, CQD/PMMA nanocomposite film can be easily peeled off from the substrate (inset in Figure 2a). The front and backside optical

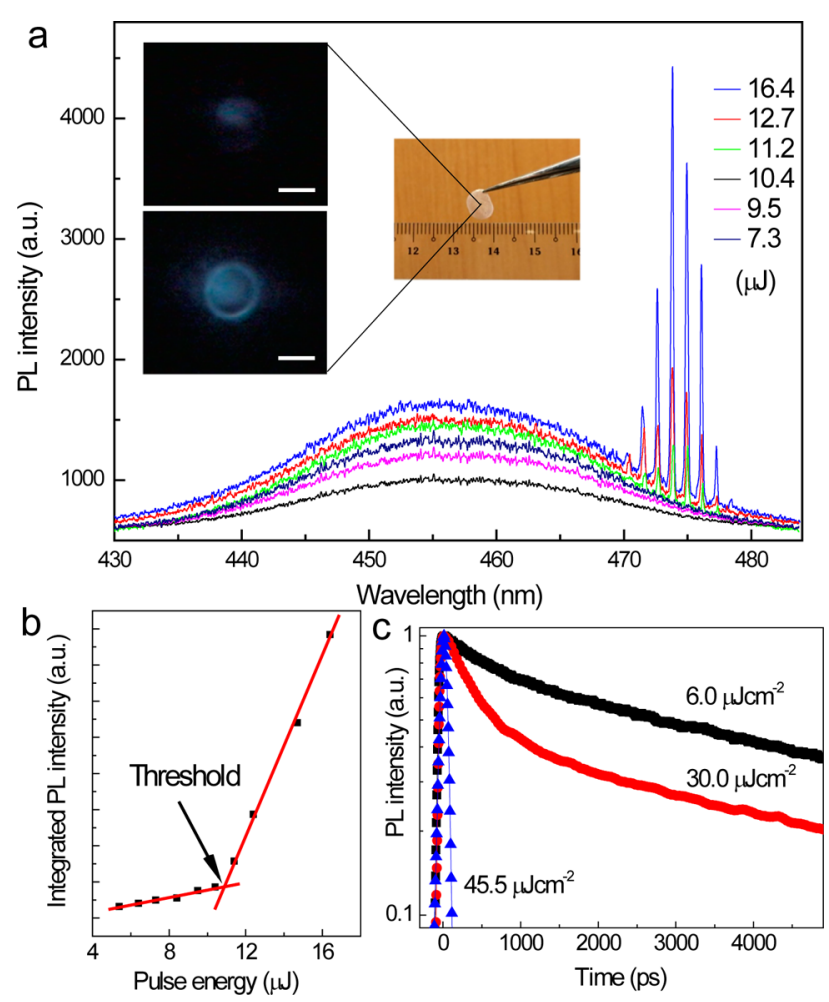

Figure 2. (a) Pump power dependent $\mu$-PL spectra from a single microbubble with diameter of $\sim 34 \mu \mathrm{m}$. The inset shows a photograph of the free-standing CQD/PMMA nanocomposite film and the PL images of the microbubble below (upper one) and above (bottom one) threshold (scale bar: $20 \mu \mathrm{m}$ ). (b) Integrated PL intensity over the sharp peak spectral range as a function of pump pulse energy. (c) Pump power dependent dynamics of the single microbubble laser.

microimages of the free-standing CQD/PMMA nanocomposite film unravel that the bubbles hang inside the polymer films (Figure S5, SI). The schematic structure of the microbubble is presented in Figure 1c.

Importantly, the self-assembled microbubbles possess nearly perfect circle boundary from top-view, which can naturally serve as WGM resonators. ${ }^{27,29,30,47}$ As a consequence, these CQDdoped microbubbles are actually potential WGM microlasers. To examine our hypothesis, we performed optical study on the individual microbubble by using a $\mu$-PL system. The excitation beam is adjusted to fully cover the individual bubble in investigation. Figure $2 \mathrm{a}$ shows the pump power dependent $\mu$ - 

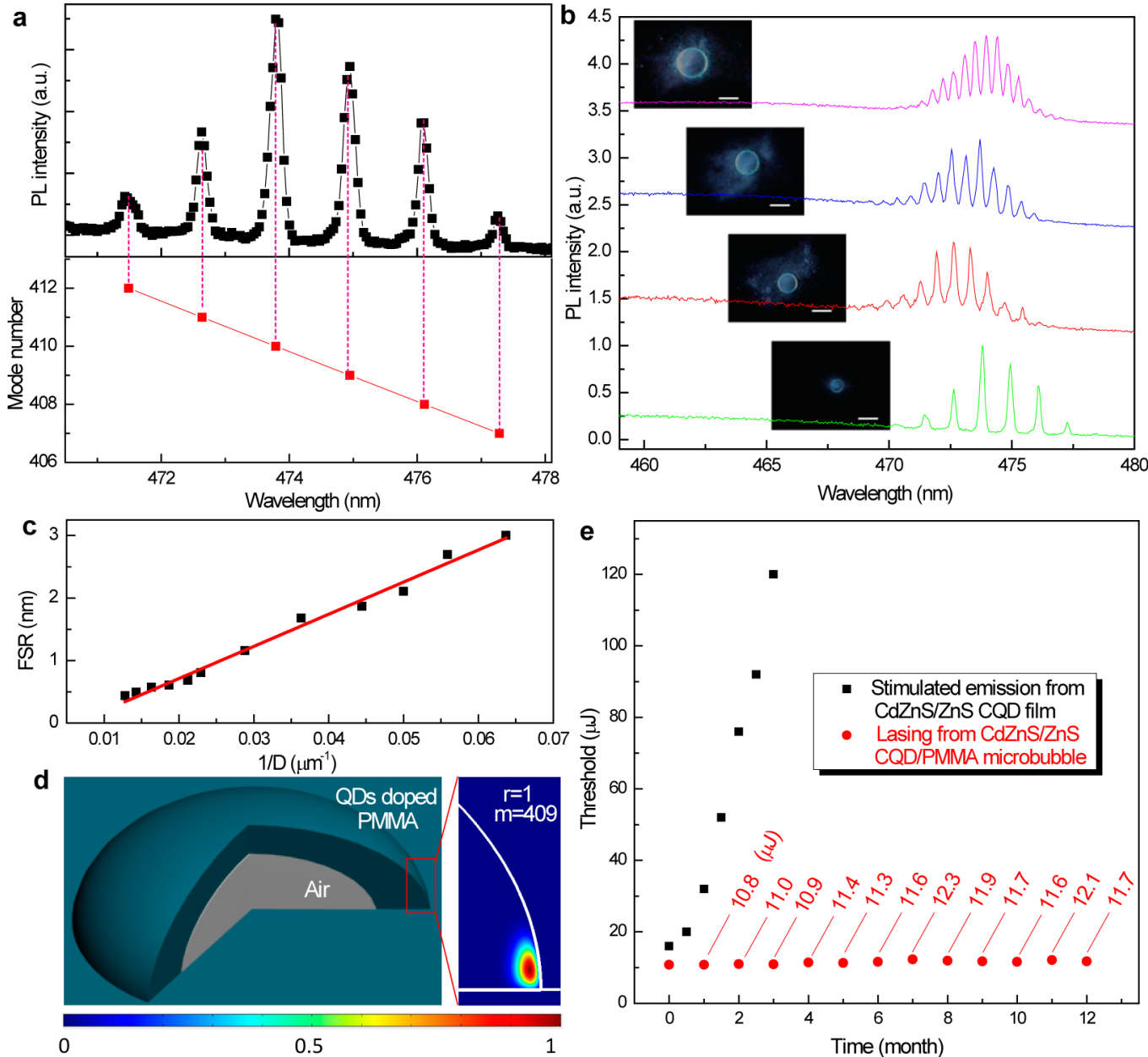

Figure 3. (a) Lasing mode assignment based on the WGM model. The red squares denote the mode numbers derived from the WGM model, which match well with the peak positions. (b) Lasing spectra from single microbubble laser with diameter of $\sim 34, \sim 47, \sim 61$, and $\sim 78 \mu \mathrm{m}$ from bottom to up (scale bar: $50 \mu \mathrm{m}$ ). (c) Plot of free spectral range (FSR) as a function of 1/D. (d) Electric field distribution in the microbubble laser. (e) Variation of stimulated emission threshold from pure $\mathrm{CdZnS/ZnS} \mathrm{CQD} \mathrm{film} \mathrm{and} \mathrm{lasing} \mathrm{threshold} \mathrm{from} \mathrm{CdZnS/ZnS} \mathrm{CQD/PMMA} \mathrm{microbubble} \mathrm{laser} \mathrm{with} \mathrm{respect}$ to storage time.

PL spectra from a single microbubble with diameter of $\sim 34 \mu \mathrm{m}$. It can be seen that, under relatively low pump pulse energies $(<10.8 \mu \mathrm{J})$, the PL spectra are dominated by the spontaneous emission. With the further increase of pump energy, sharp and comb-like discrete peaks emerge, indicating evolution from spontaneous emission to lasing action. ${ }^{10,17}$ The integrated PL intensity over the sharp peak spectral range as a function of pump pulse energy is plotted in Figure $2 b$, which reveals the development of lasing action with a low threshold of $\sim 10.8$ $\mu \mathrm{J} .{ }^{10}$ From the line-width of the lasing modes, the $Q$-factor can be derived to be as high as $\sim 2500$ based on equation: $Q=\frac{\lambda}{\Delta \lambda}$, where $\lambda$ and $\Delta \lambda$ are the peak wavelength and peak line width, respectively. ${ }^{6}$ It should be noted that the compact $Q$-switched nanosecond laser (pulse-width: 5 ns; repetition rate: $20 \mathrm{~Hz}$, wavelength: $400 \mathrm{~nm}$ ) was used as the excitation source, which is more favorable in terms of practical applications. ${ }^{10,48}$ Moreover, the pump power dependent dynamics of the single microbubble is detected by a streak camera system excited by a femtosecond laser (pulse-width: $100 \mathrm{fs}$, repetition rate: $1 \mathrm{kHz}$, wavelength: 400 $\mathrm{nm}$ ) (Figure $2 \mathrm{c}$ ). Under the low intensity of $6.0 \mu \mathrm{J} / \mathrm{cm}^{2}$, the PL decay follows that of spontaneous emission. With the increase of excitation intensity $\left(30.0 \mu \mathrm{J} / \mathrm{cm}^{2}\right)$, a fast decay path corresponding to the Auger recombination appears, which is consistent with previous reports. ${ }^{10}$ As the excitation intensity reaches $45.5 \mu \mathrm{J} /$ $\mathrm{cm}^{2}$, the emission suddenly collapses to $<50 \mathrm{ps}$, limited by the temporal resolution of the streak camera system, further confirming the development of lasing action in the CQD doped microbubble. $^{10}$

The proposed WGM lasing mechanism in the CQD microlaser can be reflected from the bright ring shape of the radiation above pump threshold (inset in Figure 2a). To be more convincing, we tentatively examine the lasing spectra according to the WGM model, where the resonant wavelength can be derived by ${ }^{49}$

$$
\begin{aligned}
\lambda=\pi n_{1} D[ & +\frac{1}{2}+2^{-1 / 3} A_{\mathrm{r}}\left(m+\frac{1}{2}\right)^{1 / 3}-\frac{q}{\sqrt{n_{\mathrm{r}}^{2}-1}} \\
& +\frac{3}{10} 2^{-2 / 3} A_{\mathrm{r}}^{2}\left(m+\frac{1}{2}\right)^{-1 / 3}-2^{-1 / 3} \\
& \left.q\left(n_{\mathrm{r}}^{2}-\frac{2}{3} q^{2}\right) \frac{A_{\mathrm{r}}\left(m+\frac{1}{2}\right)^{-2 / 3}}{\left(n_{\mathrm{r}}^{2}-1\right)^{3 / 2}}\right]^{-1}
\end{aligned}
$$


where $D$ is the diameter of the cavity, $m$ is the mode number, $n_{1}$ is the refractive index of the cavity, $n_{2}$ is the refractive index of the surrounding medium, $n_{\mathrm{r}}=\frac{n_{1}}{n_{2}}, q=n_{\mathrm{r}}$ for transverse electric (TE) mode and $q=1 / n_{r}$ for transverse magnetic (TM) mode, $r$ is the radial mode number, and $A_{\mathrm{r}}$ is the root of Airy function. We can see that the lasing peaks can be well-assigned to mode numbers indexed as 407-412 for first-order TM modes (Figure 3a), supporting the WGM lasing mechanism. Moreover, the lasing characteristics of individual microbubble with different sizes were also investigated to gain more insight (Figures S6-S8, SI). Specifically, the free spectral range (FSR) is found to decrease as the microbubble grows (Figure $3 \mathrm{~b}$ ). In a circular WGM resonator, the FSR can be calculated by ${ }^{10,47}$ FSR $=\lambda^{2} / \pi n_{\text {eff }} D$, where $n_{\text {eff }}$ is the effective refractive index of the cavity. The experimental results unambiguously disclose a linear dependence of FSR on $1 / D$ (Figure 3c), hence further confirming the WGM lasing in origin. The small-sized bubble lasers possess a large FSR, while the big-sized bubble lasers feature a higher $Q$-factor. One may determine the desired bubble size for specific applications. Finally, the electric field distribution in the microbubble laser is accessed by numerical simulation using finite element method available from COMSOL multiphysics. It is found that the field is tightly confined near the outer wall of the bubble (Figure $3 \mathrm{~d}$ and Figure S9 in the SI). Such low optical leakage owing to the total internal reflection contributes to the high $Q$-factor of the microbubble laser.

As discussed above, CQDs are generally vulnerable to ambient condition and will degrade with time. ${ }^{19}$ Figure $3 \mathrm{e}$ shows the stimulated emission threshold for the close-packed thin film of pure $\mathrm{CdZnS} / \mathrm{ZnS}$ CQDs as a function of time stored in a dark box (temperature: $\sim 20^{\circ} \mathrm{C}$; humidity: $\sim 40 \%$ ). It is found that the pump threshold gradually increases and failed to show stimulated emission after three months. Benefiting from the packaging of PMMA, the CQD-microbubble lasers exhibit unprecedentedly long-term robustness. Notably, the lasing threshold keeps almost unchanged up to one year (the same storage condition is employed as for the $\mathrm{CdZnS} / \mathrm{ZnS}$ CQD film). In addition, the CQD microlasers hold strong endurance against the tough environment. For example, the CQD microlaser can operate favorably under water (Figure S10, SI), which is very appealing for applications in photonics and biophotonics.

The facile microlaser fabrication protocol is also applicable for other kinds of CQDs such that the lasing wavelength can be broadly tuned to cover desired spectral range. As shown in Figure 4 , green and red lasing can be readily achieved in self-assembled microbubbles from green-emitting CdSSe/ZnS and red-emitting $\mathrm{CdSe} / \mathrm{CdS} \mathrm{CQD}$ doped nanocomposites. We envision that our method may be universal for all other kinds of nanoparticles with optical gain, such as upconversion nanocrystals. ${ }^{50}$

Taking advantage of the robustness, free-standing character, cost-effective and straightforward fabrication, easy mass production, and high- $Q$ these CQD microlasers hold great promise in a wide range of applications. Herein, as a proof-ofconcept, the potential of water vapor sensing based on the microbubble laser was demonstrated. In doing so, a microbubble laser with diameter of $\sim 31 \mathrm{um}$ was fixed in a glass beaker, and the beaker was covered by a transparent lid with small windows for input/output of water vapor as schematically depicted in Figure $5 c$. After filling water vapor through the input channel, the vapor source was removed immediately, and the variation of lasing spectra was dynamically monitored (Figure $5 \mathrm{a}$ ). It is found that the lasing peaks show an obvious blue-shift with the loading of
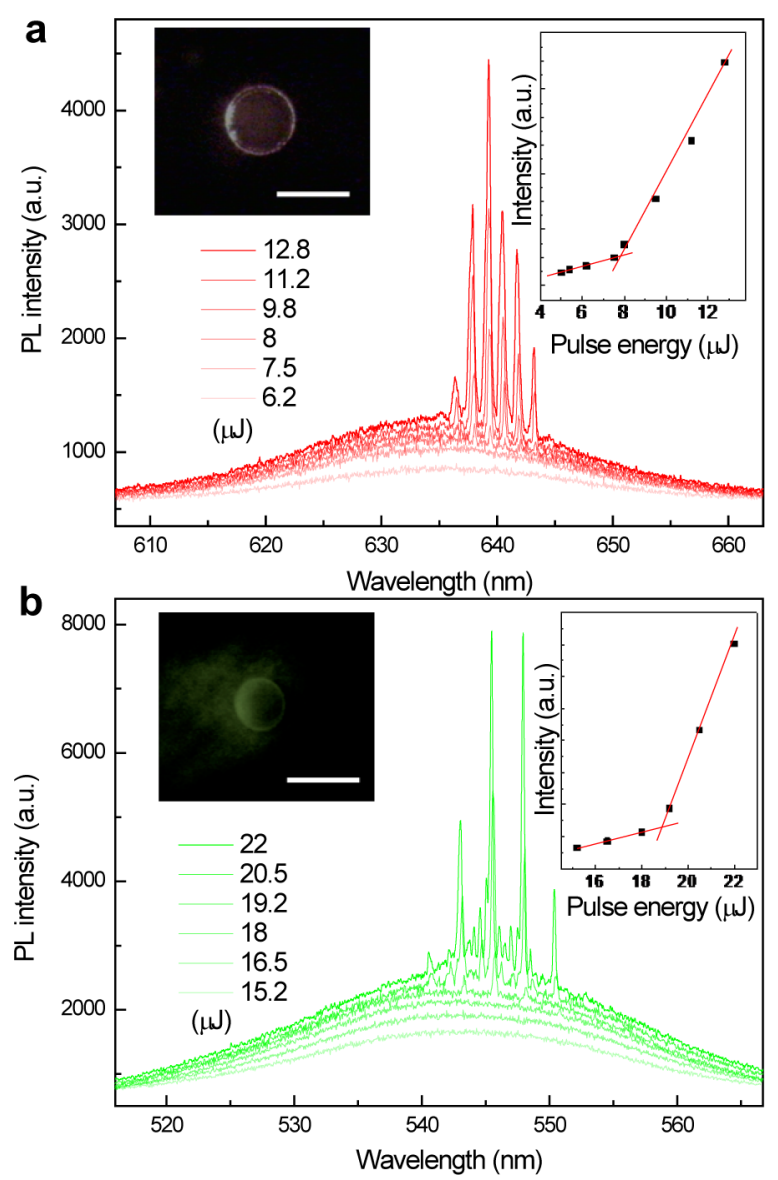

Figure 4. (a) Pump power dependent $\mu$-PL spectra from red-emitting $\mathrm{CdSe} / \mathrm{CdS} \mathrm{CQD}$ doped single microbubble, indicating the development of lasing action. The inset shows the integrated PL intensity over the sharp peak spectral range as a function of pump pulse energy and the corresponding PL image (scale bar: $50 \mu \mathrm{m}$ ). (b) Pump power dependent $\mu$-PL spectra from green-emitting CdSeS/ZnS CQD doped single microbubble, indicating the development of lasing action. The inset shows the integrated PL intensity over the sharp peak spectral range as a function of pump pulse energy and the corresponding PL image (scale bar: $50 \mu \mathrm{m}$ ).

water vapor. Such a blue-shift of the lasing modes can be attributed to the reduction of effective refractive index of PMMA with the existence of water vapor and the decrease of the diameter of the microbubble compressed by the CQD/PMMA nanocomposite base film due to the water sorption induced swelling. ${ }^{51}$ As the water vapor diffuse away from the beaker through the output channel, the lasing peaks continuously redshift (Figure 5a and $\mathrm{b}$ ) and, eventually, return to the original wavelengths before loading of water vapor, indicating the resonant mode position sensitively relates to the concentration of water vapor. Thanks to the robustness of the CQD microlaser, the reversible shift of the lasing peaks is highly reproducible under recycles of input and output of water vapor (Figure 5d). Our results unambiguously manifest that the CQD microbubble lasers are highly possible to serve as water vapor sensors given that the quantitative dependence of shifted wavelength on water vapor concentration was calibrated.

In conclusion, a novel type of microbubble WGM laser from CQD/PMMA nanocomposite was realized based on a unique but generic approach. The highly concentrated CQDs not only provide sufficient optical gain for lasing, but also assist the 

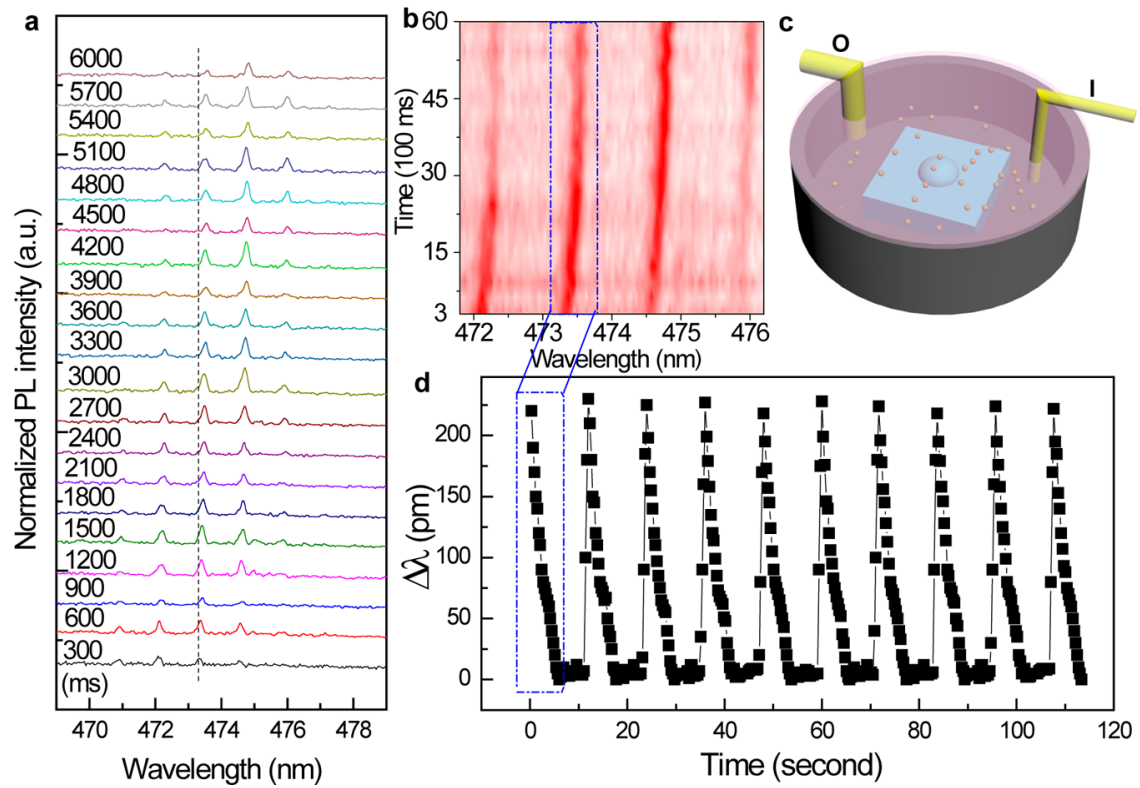

Figure 5. (a) Variation of lasing spectra from the single microbubble laser with diameter of $\sim 31$ um after loading of water vapor. (b) Lasing spectra variation mapping with time after the loading of water vapor (red color represents high intensity). (c) Setup for demonstrating water vapor sensing based on the microbubble laser (I: input; O: output). (d) Dynamic shift of lasing peak at around $473.3 \mathrm{~nm}$ with recycles of input and output of water vapor.

formation of microbubbles. These microbubbles naturally act as high-Q WGM laser resonators. Given the facile fabrication method, easy mass production, broadband lasing wavelength tunability, free-standing character, and superior robustness, these newly engineered CQD microbubble lasers would be promising in a wide range of applications including moisture sensing and biological detection.

Experimental Section. Synthesis of CdZnS/ZnS Alloyed Core-Shell CQDs. Cadmium oxide (CdO, 99.5\%), zinc acetate anhydrous ( $\mathrm{Zn}($ ace $) 2,99.99 \%)$, sulfur powder (99.98\%), 1octadecene (ODE, technical grade 90\%), and oleic acid (technical grade 90\%) were purchased from Sigma-Aldrich. Acetone was bought from Tedia, whereas methanol and toluene were purchased from Avantor Performance Materials. To synthesize the $\mathrm{CdZnS} / \mathrm{ZnS} \mathrm{CQDs}$, the one-pot hot-injection method is adopted. Briefly, a clear transparent solution of cadmium- and zinc-oleate was first acquired from a stirring mixture of $1 \mathrm{mmol}$ of $\mathrm{CdO}, 10 \mathrm{mmol}$ of $\mathrm{Zn}(\text { ace })_{2}$, and $9.5 \mathrm{~mL}$ of oleic acid, which were heated to $150{ }^{\circ} \mathrm{C}$ in a three-necked $50 \mathrm{~mL}$ round-bottom flask. After adding $12.5 \mathrm{~mL}$ ODE, the solvent was heated up to $310^{\circ} \mathrm{C}$. Then, $1.6 \mathrm{mmol}$ of sulfur dissolved in 2.4 $\mathrm{mL}$ of ODE was injected into the hot solution to initiate the nucleation. After $12 \mathrm{~min}$, the solution was cooled down, and the reaction was finished.

Optical Characterization. For micro-PL ( $\mu$-PL) experiments, the pump laser with optical wavelength of $400 \mathrm{~nm}$, pulse width of $5 \mathrm{~ns}$, and repetition rate of $20 \mathrm{~Hz}$ was guided by reflective mirrors and focused to an elliptical spot of $\sim 200 \times 300 \mu \mathrm{m}^{2}$, and an adjustable iris was placed before the focus lens to tune the spot size at will. The emission signal from the individual microbubble was gathered by a microscope objective $(50 \times, \mathrm{NA}=0.42)$. One portion of the emission was sent to a $750 \mathrm{~mm}$ monochromator and recorded by a silicon-charged coupled device (CCD), and the other portion was guided to a camera for imaging. Filters were adopted to block the residual excitation light to protect the camera and CCD. The schematic description of the setup is shown in Figure S11 in the SI. The lifetime of the samples was measured by a streak camera system with optimal temporal resolution of $\sim 50$ ps. A femtosecond laser with an optical wavelength of $400 \mathrm{~nm}$, pulse width of $100 \mathrm{fs}$, and repetition rate of $1000 \mathrm{~Hz}$ was used as the excitation source.

\section{ASSOCIATED CONTENT}

\section{S Supporting Information}

The Supporting Information is available free of charge on the ACS Publications website at DOI: 10.1021/acs.nanolett.7b00447.

Detailed information on the absorption, emission, and decay dynamics of the $\mathrm{CdZnS} / \mathrm{ZnS}$ quantum dots and the surface morphologies of the quantum dot/PMMA nanocomposite films with varied concentrations by optical microscopy and scanning electron microscopy (SEM) characterization (PDF)

Movies depicting the drying process of PMMA, CQDs, and CQD/PMMA nanocomposite in toluene (ZIP)

\section{AUTHOR INFORMATION}

\section{Corresponding Author}

*E-mail: hdsun@ntu.edu.sg.

\section{ORCID}

Hilmi Volkan Demir: 0000-0003-1793-112X

Handong Sun: 0000-0002-2261-7103

Notes

The authors declare no competing financial interest.

\section{ACKNOWLEDGMENTS}

This research is supported by the Singapore Ministry of Education through the Academic Research Fund under Projects Tier 1-RG92/15, Tier 1-RG105/16, MOE2016-T2-1-054 (S), and MOE 2011-T3-1-005 (Tier 3), and the Singapore National Research Foundation under Project No. NRFI2016-08.

\section{REFERENCES}

(1) Vahala, K. J. Nature 2003, 424 (6950), 839-846. 
(2) Xiao, Y.; Meng, C.; Wang, P.; Ye, Y.; Yu, H.; Wang, S.; Gu, F.; Dai, L.; Tong, L. Nano Lett. 2011, 11 (3), 1122-1126.

(3) Jiang, X.-F.; Xiao, Y.-F.; Zou, C.-L.; He, L.; Dong, C.-H.; Li, B.-B.; Li, Y.; Sun, F.-W.; Yang, L.; Gong, Q. Adv. Mater. 2012, 24 (35), OP260-OP264.

(4) Dang, C.; Lee, J.; Breen, C.; Steckel, J. S.; Coe-Sullivan, S.; Nurmikko, A. Nat. Nanotechnol. 2012, 7 (5), 335-339.

(5) Yakunin, S.; Protesescu, L.; Krieg, F.; Bodnarchuk, M. I.; Nedelcu, G.; Humer, M.; De Luca, G.; Fiebig, M.; Heiss, W.; Kovalenko, M. V. Nat. Commun. 2015, 6, 8056.

(6) Wang, Y.; Li, X.; Song, J.; Xiao, L.; Zeng, H.; Sun, H. Adv. Mater. 2015, 27 (44), 7101-7108.

(7) Grim, J. Q.; Manna, L.; Moreels, I. Chem. Soc. Rev. 2015, 44 (16), 5897-5914.

(8) Kovalenko, M. V.; Manna, L.; Cabot, A.; Hens, Z.; Talapin, D. V.; Kagan, C. R.; Klimov, V. I.; Rogach, A. L.; Reiss, P.; Milliron, D. J.; Guyot-Sionnnest, P.; Konstantatos, G.; Parak, W. J.; Hyeon, T.; Korgel, B. A.; Murray, C. B.; Heiss, W. ACS Nano 2015, 9 (2), 1012-1057.

(9) Klimov, V. I.; Mikhailovsky, A. A.; Xu, S.; Malko, A.; Hollingsworth, J. A.; Leatherdale, C. A.; Eisler, H. J.; Bawendi, M. G. Science 2000, 290 (5490), 314-317.

(10) Wang, Y.; Leck, K. S.; Van Duong, T.; Chen, R.; Nalla, V.; Gao, Y.; He, T.; Demir, H. V.; Sun, H. Adv. Mater. 2015, 27 (1), 169-175.

(11) Pinchetti, V.; Meinardi, F.; Camellini, A.; Sirigu, G.; Christodoulou, S.; Bae, W. K.; De Donato, F.; Manna, L.; ZavelaniRossi, M.; Moreels, I.; Klimov, V. I.; Brovelli, S. ACS Nano 2016, 10 (7), 6877-6887.

(12) Kagan, C. R.; Lifshitz, E.; Sargent, E. H.; Talapin, D. V. Science 2016, 353 (6302), 5523.

(13) Wang, Y.; Yang, S.; Yang, H.; Sun, H. Adv. Opt. Mater. 2015, 3 (5), 652-657.

(14) Adachi, M. M.; Fan, F.; Sellan, D. P.; Hoogland, S.; Voznyy, O.; Houtepen, A. J.; Parrish, K. D.; Kanjanaboos, P.; Malen, J. A.; Sargent, E. H. Nat. Commun. 2015, 6, 8694.

(15) Cooney, R. R.; Sewall, S. L.; Sagar, D. M.; Kambhampati, P. Phys. Rev. Lett. 2009, 102 (12), 127404.

(16) Jung, J.; Lin, C. H.; Yoon, Y. J.; Malak, S. T.; Zhai, Y.; Thomas, E. L.; Vardeny, V.; Tsukruk, V. V.; Lin, Z. Angew. Chem., Int. Ed. 2016, 55 (16), 5071-5075.

(17) Snee, P. T.; Chan, Y.; Nocera, D. G.; Bawendi, M. G. Adv. Mater. 2005, 17 (9), 1131-1136.

(18) Kazes, M.; Saraidarov, T.; Reisfeld, R.; Banin, U. Adv. Mater. 2009, 21 (17), 1716-1720.

(19) van Sark, W. G. J. H. M.; Frederix, P. L. T. M.; Van den Heuvel, D. J.; Gerritsen, H. C.; Bol, A. A.; van Lingen, J. N. J.; de Mello Donegá, C.; Meijerink, A. J. Phys. Chem. B 2001, 105 (35), 8281-8284.

(20) Zeng, H.; Duan, G.; Li, Y.; Yang, S.; Xu, X.; Cai, W. Adv. Funct. Mater. 2010, 20 (4), 561-572.

(21) Chan, Y.; Snee, P. T.; Caruge, J.-M.; Yen, B. K.; Nair, G. P.; Nocera, D. G.; Bawendi, M. G. J. Am. Chem. Soc. 2006, 128 (10), 31463147.

(22) Todescato, F.; Fortunati, I.; Gardin, S.; Garbin, E.; Collini, E.; Bozio, R.; Jasieniak, J. J.; Della Giustina, G.; Brusatin, G.; Toffanin, S.; Signorini, R. Adv. Funct. Mater. 2012, 22 (2), 337-344.

(23) Meinardi, F.; Colombo, A.; Velizhanin, K. A.; Simonutti, R.; Lorenzon, M.; Beverina, L.; Viswanatha, R.; Klimov, V. I.; Brovelli, S. Nat. Photonics 2014, 8 (5), 392-399.

(24) Suárez, I.; Gordillo, H.; Abargues, R.; Albert, S.; Martínez-Pastor, J. Nanotechnology 2011, 22 (43), 435202.

(25) Sun, D.; Miyatake, N.; Sue, H.-J. Nanotechnology 2007, 18 (21), 215606.

(26) Wang, Y.; Fong, K. E.; Yang, S.; Ta; Van, D.; Gao, Y.; Wang, Z.; Nalla, V.; Demir, H. V.; Sun, H. Laser Photonics Rev. 2015, 9 (5), 507516.

(27) He, L.; Özdemir, Ş. K.; Yang, L. Laser Photonics Rev. 2013, 7 (1), $60-82$.

(28) Zhang, Q.; Su, R.; Liu, X.; Xing, J.; Sum, T. C.; Xiong, Q. Adv. Funct. Mater. 2016, 26 (34), 6238-6245.
(29) Yang, S.; Wang, Y.; Sun, H. Adv. Opt. Mater. 2015, 3 (9), 11361162.

(30) Zhang, C.; Zou, C.-L.; Zhao, Y.; Dong, C.-H.; Wei, C.; Wang, H.; Liu, Y.; Guo, G.-C.; Yao, J.; Zhao, Y. S. Science Advances 2015, 1 (8), 1500257.

(31) Fan, X.; Yun, S.-H. Nat. Methods 2014, 11 (2), 141-147.

(32) Xu, C.; Dai, J.; Zhu, G.; Zhu, G.; Lin, Y.; Li, J.; Shi, Z. Laser Photonics Rev. 2014, 8 (4), 469-494.

(33) Li, B.-B.; Clements, W. R.; Yu, X.-C.; Shi, K.; Gong, Q.; Xiao, Y.-F. Proc. Natl. Acad. Sci. U. S. A. 2014, 111 (41), 14657-14662.

(34) Zhu, J.; Ozdemir, S. K.; Xiao, Y.-F.; Li, L.; He, L.; Chen, D.-R.; Yang, L. Nat. Photonics 2010, 4 (1), 46-49.

(35) Özdemir, Ş. K.; Zhu, J.; Yang, X.; Peng, B.; Yilmaz, H.; He, L.; Monifi, F.; Huang, S. H.; Long, G. L.; Yang, L. Proc. Natl. Acad. Sci. U. S. A. 2014, 111 (37), E3836-E3844.

(36) He, L.; Ozdemir, S. K.; Zhu, J.; Kim, W.; Yang, L. Nat. Nanotechnol. 2011, 6 (7), 428-432.

(37) Min, B.; Kim, S.; Okamoto, K.; Yang, L.; Scherer, A.; Atwater, H.; Vahala, K. Appl. Phys. Lett. 2006, 89 (19), 191124.

(38) Grivas, C.; Li, C.; Andreakou, P.; Wang, P.; Ding, M.; Brambilla, G.; Manna, L.; Lagoudakis, P. Nat. Commun. 2013, 4, 2376.

(39) Peng, S.; Xu, C.; Hughes, T. C.; Zhang, X. Langmuir 2014, 30 (41), 12270-12277.

(40) Epstein, P. S.; Plesset, M. S. J. Chem. Phys. 1950, 18 (11), 15051509.

(41) Ljunggren, S.; Eriksson, J. C. Colloids Surf., A 1997, 129-130, 151155.

(42) Peng, S.; Mega, T. L.; Zhang, X. Langmuir 2016, 32 (43), 1126511272.

(43) Lohse, D.; Zhang, X. Rev. Mod. Phys. 2015, 87 (3), 981-1035.

(44) Evchuk, I. Y.; Musii, R. I.; Makitra, R. G.; Pristanskii, R. E. Russ. J. Appl. Chem. 2005, 78 (10), 1576-1580.

(45) Ljunggren, S.; Eriksson, J. C.; Kralchevsky, P. A. J. Colloid Interface Sci. 1997, 191 (2), 424-441.

(46) Lin, H.-J. Study and applications of hybrid organic/ inorganic semiconductor quantum dots in thin films; Aix-Marseille University, Optics/Photonics, 2013.

(47) Ta, V. D.; Chen, R.; Sun, H. D. Adv. Mater. 2012, 24 (10), OP60OP64.

(48) Oron, D.; Kazes, M.; Banin, U. Phys. Rev. B: Condens. Matter Mater. Phys. 2007, 75 (3), 035330.

(49) Yang, S.; Ta, V. D.; Wang, Y.; Chen, R.; He, T.; Demir, H. V.; Sun, H. Sci. Rep. 2016, 6, 27200.

(50) Zhu, H.; Chen, X.; Jin, L. M.; Wang, Q. J.; Wang, F.; Yu, S. F. ACS Nano 2013, 7 (12), 11420-11426.

(51) Watanabe, T.; Ooba, N.; Hida, Y.; Hikita, M. Appl. Phys. Lett. 1998, 72 (13), 1533-1535. 